\title{
Association Between Self-Reported Bruxism and Malocclusion in University Students: A Cross-Sectional Study
}

\author{
Kota Kataoka ${ }^{1}$, Daisuke Ekuni ${ }^{1}$, Shinsuke Mizutani ${ }^{1}$, Takaaki Tomofuji ${ }^{1}, 2$, Tetsuji Azuma ${ }^{1}$, \\ Mayu Yamane ${ }^{1}$, Yuya Kawabata ${ }^{1}$, Yoshiaki Iwasaki ${ }^{3}$, and Manabu Morita ${ }^{1}$
}

${ }^{1}$ Department of Preventive Dentistry, Okayama University Graduate School of Medicine, Dentistry and Pharmaceutical Sciences, Okayama, Japan

${ }^{2}$ Advanced Research Center for Oral and Craniofacial Sciences, Okayama University Dental School, Okayama, Japan

${ }^{3}$ Health Service Center, Okayama University, Okayama, Japan

Received October 1, 2014; accepted January 7, 2015; released online April 11, 2015

Copyright (C) 2015 Kota Kataoka et al. This is an open access article distributed under the terms of Creative Commons Attribution License, which permits unrestricted use, distribution, and reproduction in any medium, provided the original author and source are credited.

\begin{abstract}
Objectives: Bruxism can result in temporomandibular disorders, oral pain, and tooth wear. However, it is unclear whether bruxism affects malocclusion. The aim of this study was to examine the association between self-reported bruxism and malocclusion in university students.

Methods: Students ( $n=1503 ; 896$ men and 607 women) aged 18 and 19 years were examined. Malocclusion was defined using a modified version of the Index of Orthodontic Treatment Need. The presence of buccal mucosa ridging, tooth wear, dental impression on the tongue, palatal/mandibular torus, and the number of teeth present were recorded, as well as body mass index (BMI). Additional information regarding gender, awareness of bruxism, orthodontic treatment, and oral habits was collected via questionnaire.

Results: The proportion of students with malocclusion was $32 \%(n=481)$. The awareness of clenching in males with malocclusion was significantly higher than in those with normal occlusion (chi square test, $P<0.01$ ). According to logistic regression analysis, the probability of malocclusion was significantly associated with awareness of clenching (odds ratio [OR] 2.19; 95\% confidence interval [CI], 1.22-3.93) and underweight (BMI $<18.5 \mathrm{~kg} / \mathrm{m}^{2}$ ) (OR 1.89; 95\% CI, 1.31-2.71) in males but not in females. In subgroup analyses, the probability of crowding was also significantly associated with awareness of clenching and underweight $(P<0.01)$ in males.

Conclusions: Awareness of clenching and underweight were related to malocclusion (crowding) in university male students.
\end{abstract}

Key words: bruxism; malocclusion; young adult; cross-sectional studies

\section{INTRODUCTION}

Bruxism, defined as the parafunctional grinding of teeth, is an oral habit consisting of involuntary rhythmic or spasmodic non-functional gnashing, grinding, or clenching of the teeth other than chewing movements of the mandible. ${ }^{1}$ Although this definition is commonly used by dental professionals, there is no link to the sleep-wake state of the condition. ${ }^{2}$ Sleep bruxism is clearly defined as 'an oral parafunction characterized by grinding or clenching of the teeth during sleep that is associated with excessive sleep arousal activity'. ${ }^{3}$ However, awake bruxism still lacks a clear definition. ${ }^{2}$

The prevalence of bruxism ranges widely, from $4 \%$ to $96 \%,{ }^{4-10}$ because of differences in the bruxism types (unspecified, sleep, and awake), applied diagnostic methodology (questionnaires, oral history, and clinical examination), the presence or absence of comorbidities (eg, anxiety or temporomandibular disorder), and the characteristics of the study population. ${ }^{2}$ The prevalence of bruxism is higher in young adults than in the elderly. ${ }^{6,11,12}$ The etiology of bruxism remains controversial. Recent reviews suggest that bruxism is mainly regulated by pathophysiological and psychological factors, rather than morphological ones. ${ }^{13,14}$ Although some dentists suggest that malocclusion may cause bruxism, a recent review concluded that there is no evidence whatsoever for a causal relationship between bruxism and occlusion. ${ }^{2}$ Thus, the research focus is mainly on psychosocial, ${ }^{15,16}$ physiological/biological, ${ }^{17-20}$ and exogenous factors. ${ }^{21}$ However, the etiological factors for bruxism are still unclear, and the etiology is probably multifactorial. ${ }^{15-22}$ 
Intermittent bruxism, including clenching and grinding, is extremely common, but it usually poses no serious consequences for the oral structures. On the other hand, frequent bruxism can result in problems. Possible sequelae of bruxism include tooth wear, signs and symptoms of temporomandibular disorders, headache, toothache, mobile teeth, and various problems with dental restorations as well as with fixed and removable prostheses. ${ }^{5}$ However, whether possible consequences of bruxism also include malocclusion remains unclear.

Malocclusion is a developmental disorder of the maxillofacial system, and inflicts functional and esthetic disturbances. Malocclusion, including crowding, open bite, overjet, and crossbite, is associated with psychological stress as well as impaired oral health. ${ }^{23}$ The etiology of malocclusion has genetic and environmental components, ${ }^{24}$ and study of malocclusion is essential for the success of orthodontic treatment, since a prerequisite for correction is the elimination of the causes of malocclusion. ${ }^{25}$ Analysis of factors related to the causes of malocclusion is important for planning public health policies aimed at preventing and clinically intercepting the health problem. ${ }^{26}$ Since excessive force exerted by bruxism can lead to tooth movement, ${ }^{27}$ bruxism may result in malocclusion. Therefore, we hypothesized that bruxism may be a risk factor of malocclusion. The aim of this study was to investigate the association between bruxism and malocclusion in university students.

\section{MATERIALS AND METHODS}

\section{Study population}

Of 2303 first-year students who underwent a general health examination at the Health Service Center of Okayama University in April 2013, 2205 students volunteered to receive an oral examination and answered the questionnaire described below. The general health examination is mandatory for first-year students in all departments in the university. We excluded 702 participants who were $\geq 20$ years old ( $n=113$ ), who had received or were currently undergoing orthodontic treatment $(n=431)$, or who had provided incomplete data in their questionnaires $(n=158)$. Therefore, data from 1503 students (896 men and 607 women) were analyzed. The study was approved by the Ethics Committee of Okayama University Graduate School of Medicine, Dentistry and Pharmaceutical Sciences. Written consent was obtained from all participants.

\section{Questionnaire}

We mailed a questionnaire to the participants before the health examination. Besides gender, age, and general condition, the questionnaire included questions on awareness of bruxism and oral habits. Questions to identify awareness of bruxism included the following: During the past 3 months, 1) Has anyone heard you grinding your teeth at night?; 2) Is your jaw ever fatigued or sore on waking in the morning?; 3) Are your teeth or gums ever sore on waking in the morning?; 4) Do you ever experience temporal headache on waking in the morning?; 5) Are you ever aware of grinding your teeth during the day?; and 6) Are you ever aware of clenching your teeth during the day? ${ }^{3,28-30}$ Each question was answered by selecting a frequency (frequently, sometimes, rarely, or never). We combined "frequently" and "sometimes" responses into a single category of positive awareness and rarely and never responses into a single category of negative awareness. The validity and reliability of the questionnaire has been already confirmed as useful for evaluating bruxism. ${ }^{3,28-30}$ For oral habits, answers were given by participants in a "yes/no" format as follows: biting fingernail/pens/pencils, biting mucosa of cheeks/lips, and gum chewing. ${ }^{31-33}$

\section{Assessment of body mass index}

In the general health examination, the height and body weight of participants were measured by the university's public health nurses using the Tanita body fat analyser (Model No. BF-220; Tanita Co., Tokyo, Japan). Since body mass index (BMI) may be related to jaw growth, BMI was computed as weight in kilograms divided by height in meters squared. ${ }^{34}$ For this analysis, categories of BMI were calculated based on the accepted cut-off values of underweight (BMI $<18.5 \mathrm{~kg} / \mathrm{m}^{2}$ ), normal weight $\left(18.5-24.9 \mathrm{~kg} / \mathrm{m}^{2}\right)$, overweight $(25-29.9$ $\left.\mathrm{kg} / \mathrm{m}^{2}\right)$, and obese $\left(\geq 30 \mathrm{~kg} / \mathrm{m}^{2}\right) .{ }^{35}$

\section{Oral examination}

Five dentists (S.M., D.E., T.A., M.Y., and K.K.) examined the study participants. In addition to the number of teeth present, occlusal tooth wear was assessed using the occlusal tooth wear index: score $0=$ no loss of occlusal enamel surface characteristics; score $1=$ loss of occlusal enamel surface characteristics; score $2=$ loss of occlusal enamel, exposing dentine for less than $1 / 3$ of the surface/incisal loss of enamel and minimal dentine exposure; score $3=$ loss of occlusal enamel, exposing dentine for more than $1 / 3$ of the surface/ incisal loss of enamel and substantial loss of dentine; and score $4=$ complete loss of occlusal/incisal enamel, pulp exposure or exposure of secondary dentine. ${ }^{36}$

For malocclusion, a modified version of the Index of Orthodontic Treatment Need (IOTN) was used for each participant. ${ }^{23}$ The modified IOTN that we used does not define a definite aesthetic need for treatment (AC grades 8, 9, and 10). However, previous studies suggested that our modified version of IOTN without a definite aesthetic need for treatment, ${ }^{23,37}$ as well as the modified IOTN (the original reference $)^{38}$ are useful for screening malocclusion by nonspecialists in oral health surveys. The dental health component of the modified IOTN consists of a two-grade scale $(0=$ no definite need for orthodontic treatment [normal occlusion group] and $1=$ definite need for orthodontic treatment [malocclusion group]). Further, for subgroup analysis, the 
type of malocclusion (crowding, overjet, overbite, crossbites, and missing teeth) was recorded using a CPI probe (YDM, Tokyo, Japan).

The diagnostic criterion of buccal mucosa ridging or tongue indentation was defined as a linear thickening at the level where the teeth occlude on the buccal mucosa or tongue, respectively. ${ }^{30}$ In buccal mucosa ridging, the range (none, partial, and widespread) was also evaluated.

The presence or absence of palatal torus and mandibular torus was examined. ${ }^{39}$ Palatal torus was assessed as present when a painless bony swelling was visualized or palpated in the middle of the hard palate. ${ }^{40}$ Mandibular torus was assessed as present when a painless bony outgrowth in the lingual area of the mandible was visualized or palpated.

In a preliminary check, each kappa value (for occlusal tooth wear, malocclusion, buccal mucosa ridging, dental impression on the tongue, palatal torus, and mandibular torus) was more than 0.80 .

\section{Statistical analyses}

Unpaired $t$ or chi-squared tests were used to determine whether there were any significant differences $(P<0.05)$ between men and women and between the normal occlusion and malocclusion groups. Odds ratios (ORs) and 95\% confidence intervals (CIs) were calculated using a series of logistic regression models. Presence of malocclusion or crowding was used as a dependent variable. Based on the binary analyses, BMI category and clenching during the day were added as independent variables on multivariate analysis. In the multivariate analyses, we excluded tooth wear, buccal mucosa ridging, tongue indentation, palatal torus, and mandibular torus from the independent valuables because most of these items still contain vague factors and the validity has not been confirmed. ${ }^{30,41}$ A statistical program (PASW version 18.0; IBM, Tokyo, Japan) was used for statistical analyses.

\section{RESULTS}

Table 1 shows the characteristics of participants. It was observed that $32.0 \%$ of participants had malocclusion $(n=481)$, with the prevalence of malocclusion in females being significantly higher than that in males $(P<0.05)$. Since there were significant differences in other clinical parameters, awareness of bruxism, and oral habits between males and females $(P<0.05)$, further analyses were performed in each gender.

Table 2 shows the association between malocclusion and related factors in males. There were significant differences in awareness of daytime clenching, buccal mucosa ridging, tongue indentation, mandibular torus, and BMI between the malocclusion and normal occlusion groups $(P<0.05)$.

In females, there were significant differences in maximum occlusal tooth wear index score, buccal mucosa ridging,
Table 1. Characteristics of participants

\begin{tabular}{|c|c|c|c|c|}
\hline Parameter & $\begin{array}{l}\text { Males } \\
(n=896)\end{array}$ & $\begin{array}{l}\text { Females } \\
(n=607)\end{array}$ & $\begin{array}{c}\text { Total } \\
(n=1503)\end{array}$ & $P$ value $^{\mathrm{a}}$ \\
\hline BMI $\left(\mathrm{kg} / \mathrm{m}^{2}\right)$ & $21.2 \pm 3.1$ & $20.5 \pm 2.5$ & $20.9 \pm 2.9$ & $<0.001$ \\
\hline BMI category & & & & 0.001 \\
\hline Underweight $\left(\mathrm{BMI}<18.5 \mathrm{~kg} / \mathrm{m}^{2}\right.$ ) & $157(17.5)$ & $130(21.4)$ & $287(19.1)$ & \\
\hline Normal weight (BMI $18.5-24.9 \mathrm{~kg} / \mathrm{m}^{2}$ ) & $651(72.7)$ & $450(74.1)$ & 1101 (73.3) & \\
\hline Overweight (BMI 25-29.9kg/m²) & $78(8.7)$ & $23(3.8)$ & $101(6.7)$ & \\
\hline Obesity (BMI $\geq 30 \mathrm{~kg} / \mathrm{m}^{2}$ ) & $10(1.1)$ & $4(0.7)$ & $14(0.9)$ & \\
\hline Number of teeth present & $28.7 \pm 1.3$ & $28.4 \pm 1.1$ & $28.5 \pm 1.3$ & $<0.001$ \\
\hline Maximum occlusal tooth wear index score & & & & 0.007 \\
\hline 0 & $211(23.5)$ & $189(31.1)$ & $400(26.6)$ & \\
\hline 1 & 499 (55.7) & $297(48.9)$ & $796(53.0)$ & \\
\hline 2 & $168(18.8)$ & $105(17.3)$ & $273(18.2)$ & \\
\hline 3 & $18(2.0)$ & $16(2.6)$ & $34(2.3)$ & \\
\hline 4 & $0(0.0)$ & $0(0.0)$ & $0(0.0)$ & \\
\hline Buccal mucosa ridging & & & & $<0.001$ \\
\hline No & $379(42.3)$ & $178(29.3)$ & $557(37.1)$ & \\
\hline Partial & $380(42.4)$ & $320(52.7)$ & $700(46.6)$ & \\
\hline Widespread & $137(15.3)$ & $109(18.0)$ & $246(16.4)$ & \\
\hline Tongue indentation & $249(27.8)$ & $207(34.1)$ & $456(30.3)$ & 0.009 \\
\hline Palatal torus & $6(0.7)$ & $14(2.3)$ & $20(1.3)$ & 0.007 \\
\hline Mandibular torus & $146(16.3)$ & $92(15.2)$ & $238(15.8)$ & 0.553 \\
\hline Malocclusion & $268(29.9)$ & $213(35.1)$ & $481(32.0)$ & 0.025 \\
\hline Crowding & $191(21.3)$ & $142(23.4)$ & $333(22.2)$ & 0.341 \\
\hline Overjet & $67(7.5)$ & $56(9.2)$ & $123(8.2)$ & 0.102 \\
\hline Overbite & $5(0.6)$ & $1(0.2)$ & $6(0.4)$ & 0.235 \\
\hline Crossbites & $20(2.2)$ & $14(2.3)$ & $34(2.3)$ & 0.924 \\
\hline Missing teeth & $18(2.0)$ & $7(1.2)$ & $25(1.7)$ & 0.203 \\
\hline \multicolumn{5}{|l|}{ Awareness of bruxism } \\
\hline Jaw fatigue on waking in the morning & $23(2.6)$ & $21(3.5)$ & $44(2.9)$ & 0.314 \\
\hline $\begin{array}{l}\text { Sore teeth or gums on waking } \\
\text { in the morning }\end{array}$ & $20(2.2)$ & $16(2.6)$ & $36(2.4)$ & 0.615 \\
\hline Headache on waking in the morning & $57(6.4)$ & $52(8.6)$ & $109(7.3)$ & 0.106 \\
\hline Grinding during the day & $13(1.5)$ & $20(3.3)$ & $33(2.2)$ & 0.017 \\
\hline Clenching during the day & $49(5.5)$ & $76(12.5)$ & $125(8.3)$ & $<0.001$ \\
\hline Sleep bruxism & $49(5.5)$ & $52(8.6)$ & $101(6.7)$ & 0.019 \\
\hline \multicolumn{5}{|l|}{ Oral habits } \\
\hline Biting fingernail/pens/pencils & $91(10.2)$ & $39(6.4)$ & $130(8.6)$ & 0.012 \\
\hline Biting mucosa of cheeks/lips & $164(18.3)$ & $114(18.8)$ & $278(18.5)$ & 0.815 \\
\hline Gum chewing & $134(15.0)$ & $46(7.6)$ & $180(12.0)$ & $<0.001$ \\
\hline
\end{tabular}

BMI, body mass index.

All values are reported as number (\%) except for BMI, which is reported as mean \pm standard deviation.

at test or $\chi^{2}$ test.

tongue indentation, palatal torus, and mandibular torus between the malocclusion and normal occlusion groups $(P<0.05)$ (Table 3). However, significant between-group differences in awareness of daytime clenching and BMI were not observed in females.

On logistic regression analyses, the risk of malocclusion was significantly related to underweight $\left(\mathrm{BMI}<18.5 \mathrm{~kg} / \mathrm{m}^{2}\right)$ and clenching during the day in males $(P<0.01)$ (Table 4$)$. However, there was no significant association between malocclusion and any of the parameters in females.

Because of the small number of participants with overbite, crossbites, and missing teeth, further subgroup analysis was performed only between normal occlusion and crowding groups and between normal occlusion and overjet groups.

Table 5 shows the association between crowding and related factors in males. There were significant differences in BMI, buccal mucosa ridging, tongue indentation, awareness of daytime clenching, and mandibular torus between the crowding and normal occlusion groups $(P<0.05)$.

In females, there were significant differences in number of teeth present, maximum occlusal tooth wear index score, buccal mucosa ridging, tongue indentation, palatal torus, and mandibular torus between the crowding and normal occlusion 
Table 2. Association between malocclusion and related factors in males

\begin{tabular}{|c|c|c|c|}
\hline Parameter & $\begin{array}{c}\text { Normal occlusion } \\
\quad(n=628)\end{array}$ & $\begin{array}{c}\text { Malocclusion } \\
\quad(n=268)\end{array}$ & $P$ value \\
\hline $\mathrm{BMI}\left(\mathrm{kg} / \mathrm{m}^{2}\right)$ & $21.3 \pm 3.1$ & $20.8 \pm 3.0$ & 0.022 \\
\hline BMI category & & & 0.006 \\
\hline Underweight $\left(\mathrm{BMI}<18.5 \mathrm{~kg} / \mathrm{m}^{2}\right)$ & $92(14.6)$ & 65 (24.3) & \\
\hline Normal weight (BMI $18.5-24.9 \mathrm{~kg} / \mathrm{m}^{2}$ ) & $473(75.3)$ & $178(66.4)$ & \\
\hline Overweight (BMI $25-29.9 \mathrm{~kg} / \mathrm{m}^{2}$ ) & $55(8.8)$ & $23(8.6)$ & \\
\hline Obesity (BMI $\geq 30 \mathrm{~kg} / \mathrm{m}^{2}$ ) & $8(1.3)$ & $2(0.7)$ & \\
\hline Number of teeth present & $28.7 \pm 1.3$ & $28.6 \pm 1.3$ & 0.398 \\
\hline Maximum occlusal tooth wear index score & & & 0.062 \\
\hline 0 & $150(23.9)$ & $61(22.8)$ & \\
\hline 1 & $358(57.0)$ & $141(52.6)$ & \\
\hline 2 & $112(17.8)$ & $56(20.9)$ & \\
\hline 3 & $8(1.3)$ & $10(3.7)$ & \\
\hline 4 & $0(0.0)$ & $0(0.0)$ & \\
\hline Buccal mucosa ridging & & & $<0.001$ \\
\hline No & $295(47.0)$ & $84(31.3)$ & \\
\hline Partial & $249(39.6)$ & $131(48.9)$ & \\
\hline Widespread & $84(13.4)$ & $53(19.8)$ & \\
\hline Tongue indentation & $157(25.0)$ & $92(34.3)$ & 0.004 \\
\hline Palatal torus & $4(0.6)$ & $2(0.7)$ & 0.854 \\
\hline Mandibular torus & $80(12.7)$ & $66(24.6)$ & $<0.001$ \\
\hline \multicolumn{4}{|l|}{ Awareness of bruxism } \\
\hline Jaw fatigue on waking in the morning & $15(2.4)$ & $8(3.0)$ & 0.605 \\
\hline Sore teeth or gums on waking in the morning & $12(1.9)$ & $8(3.0)$ & 0.319 \\
\hline Headache on waking in the morning & $46(7.3)$ & $11(4.1)$ & 0.071 \\
\hline Grinding during the day & $10(1.6)$ & $3(1.1)$ & 0.588 \\
\hline Clenching during the day & $26(4.1)$ & $23(8.6)$ & 0.007 \\
\hline Sleep bruxism & $34(5.4)$ & $15(5.6)$ & 0.912 \\
\hline \multicolumn{4}{|l|}{ Oral habits } \\
\hline Biting fingernail/pens/pencils & $67(10.7)$ & $24(9.0)$ & 0.437 \\
\hline Biting mucosa of cheeks/lips & $106(16.9)$ & $58(21.6)$ & 0.091 \\
\hline Gum chewing & $96(15.3)$ & $38(14.2)$ & 0.670 \\
\hline
\end{tabular}

BMl, body mass index.

All values are reported as number (\%) except for BMI, which is reported as mean \pm standard deviation.

${ }^{a} t$ test or $\chi^{2}$ test.

groups $(P<0.05)$ (Table 6). However, significant betweengroup differences in awareness of daytime clenching and BMI were not observed in females.

On logistic regression analyses, the risk of crowding was significantly related to underweight $\left(B M I<18.5 \mathrm{~kg} / \mathrm{m}^{2}\right)$ and clenching during the day in males $(P<0.01)$ (Table 7). In females, the risk of crowding was significantly related to the number of teeth present $(P<0.01)$ (Table 7$)$.

Table 8 and Table 9 show the association between overjet and related factors in males and females, respectively. There were no significant differences in any factor between the overjet and normal occlusion groups $(P>0.05)$.

\section{DISCUSSION}

This is the first large-scale cross-sectional study to examine the association between malocclusion and awareness of bruxism in young adults based on the new hypothesis that bruxism may contribute to malocclusion. The results revealed that the risk of malocclusion, especially crowding, was significantly related to clenching during the day in males. Because the force of bruxism can lead to tooth movement ${ }^{27}$ and the tightness of proximal tooth contact is increased by clenching, ${ }^{42}$ clenching during the day may be a risk factor of malocclusion (crowding) through tooth movement in males.
Table 3. Association between malocclusion and related factors in females

\begin{tabular}{|c|c|c|c|}
\hline Parameter & $\begin{array}{l}\text { Normal occlusion } \\
\quad(n=394)\end{array}$ & $\begin{array}{l}\text { Malocclusion } \\
\quad(n=213)\end{array}$ & $P$ value $^{\mathrm{a}}$ \\
\hline $\mathrm{BMI}\left(\mathrm{kg} / \mathrm{m}^{2}\right)$ & $20.5 \pm 2.4$ & $20.5 \pm 2.7$ & 0.990 \\
\hline BMI category & & & 0.252 \\
\hline Underweight (BMI $<18.5 \mathrm{~kg} / \mathrm{m}^{2}$ ) & $81(20.6)$ & $49(23.0)$ & \\
\hline Normal weight (BMI 18.5-24.9kg/m²) & $295(74.9)$ & $155(72.8)$ & \\
\hline Overweight (BMI 25-29.9kg/m²) & $17(4.3)$ & $6(2.8)$ & \\
\hline Obesity (BMI $\geq 30 \mathrm{~kg} / \mathrm{m}^{2}$ ) & $1(0.3)$ & $3(1.4)$ & \\
\hline Number of teeth present & $28.4 \pm 1.1$ & $28.5 \pm 1.2$ & 0.369 \\
\hline Maximum occlusal tooth wear index score & & & 0.014 \\
\hline 0 & $114(28.9)$ & $75(35.3)$ & \\
\hline 1 & $208(52.8)$ & $89(41.8)$ & \\
\hline 2 & $66(16.8)$ & $39(18.3)$ & \\
\hline 3 & $6(1.5)$ & $10(4.7)$ & \\
\hline 4 & $0(0.0)$ & $0(0.0)$ & \\
\hline Buccal mucosa ridging & & & 0.002 \\
\hline No & $134(34.0)$ & $44(20.7)$ & \\
\hline Partial & $190(48.2)$ & $130(61.0)$ & \\
\hline Widespread & $70(17.8)$ & 39 (18.3) & \\
\hline Tongue indentation & $118(29.9)$ & 89 (41.8) & 0.003 \\
\hline Palatal torus & $4(1.0)$ & $10(4.7)$ & 0.004 \\
\hline Mandibular torus & $38(9.6)$ & $54(25.4)$ & $<0.001$ \\
\hline \multicolumn{4}{|l|}{ Awareness of bruxism } \\
\hline Jaw fatigue on waking in the morning & $13(3.3)$ & $8(3.8)$ & 0.769 \\
\hline Sore teeth or gums on waking in the morning & $10(2.5)$ & $6(2.8)$ & 0.838 \\
\hline Headache on waking in the morning & $33(8.4)$ & $19(8.9)$ & 0.819 \\
\hline Grinding during the day & $11(2.8)$ & $9(4.2)$ & 0.345 \\
\hline Clenching during the day & $50(12.7)$ & $26(12.2)$ & 0.864 \\
\hline Sleep bruxism & $37(9.4)$ & $15(7.0)$ & 0.324 \\
\hline \multicolumn{4}{|l|}{ Oral habits } \\
\hline Biting fingernail/pens/pencils & $25(6.3)$ & $14(6.6)$ & 0.913 \\
\hline Biting mucosa of cheeks/lips & $82(20.8)$ & $32(15.0)$ & 0.081 \\
\hline Gum chewing & $28(7.1)$ & $18(8.5)$ & 0.550 \\
\hline
\end{tabular}

BMI, body mass index.

All values are reported as number (\%) except for BMI, which is reported as mean \pm standard deviation.

${ }^{\text {a }} t$ test or $\chi^{2}$ test.

Table 4. Adjusted odds ratios and $95 \%$ confidence intervals for malocclusion

\begin{tabular}{|c|c|c|c|c|}
\hline Parameter & & Odds ratio & $95 \% \mathrm{Cl}^{\mathrm{a}}$ & $P$ value \\
\hline \multicolumn{5}{|l|}{ Males } \\
\hline \multirow[t]{3}{*}{ BMI category } & $\begin{array}{l}\text { Normal weight } \\
\left(\text { BMl } 18.5-24.9 \mathrm{~kg} / \mathrm{m}^{2}\right)\end{array}$ & 1 & & \\
\hline & $\begin{array}{l}\text { Underweight } \\
\left(\mathrm{BMl}<18.5 \mathrm{~kg} / \mathrm{m}^{2}\right)\end{array}$ & 1.89 & $1.31-2.71$ & 0.001 \\
\hline & $\begin{array}{l}\text { Overweight/obesity } \\
\left(\mathrm{BMI} \geq 25 \mathrm{~kg} / \mathrm{m}^{2}\right)\end{array}$ & 1.08 & $0.66-1.77$ & 0.771 \\
\hline Clenching & - & 1 & & \\
\hline during the day & + & 2.19 & $1.22-3.93$ & 0.009 \\
\hline \multicolumn{5}{|l|}{ Females } \\
\hline \multirow[t]{3}{*}{ BMI category } & $\begin{array}{l}\text { Normal weight } \\
\left(\text { BMl } 18.5-24.9 \mathrm{~kg} / \mathrm{m}^{2}\right)\end{array}$ & 1 & & \\
\hline & $\begin{array}{l}\text { Underweight } \\
\left(\mathrm{BMl}<18.5 \mathrm{~kg} / \mathrm{m}^{2}\right)\end{array}$ & 1.17 & $0.78-1.76$ & 0.438 \\
\hline & $\begin{array}{l}\text { Overweight/obesity } \\
\left.\text { (BMI } \geq 25 \mathrm{~kg} / \mathrm{m}^{2}\right)\end{array}$ & 0.94 & $0.41-2.15$ & 0.888 \\
\hline Clenching & - & 1 & & \\
\hline during the day & + & 1.06 & $0.64-1.74$ & 0.833 \\
\hline
\end{tabular}

$\mathrm{BMI}$, body mass index; $\mathrm{Cl}$, confidence interval.

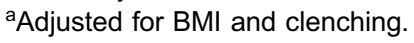

The relationship between bruxism and occlusion has been investigated in dentistry for a long time but has remained poorly understood. ${ }^{2}$ Although some dentists suggest that malocclusion may cause bruxism, a recent review concluded 
Table 5. Association between crowding and related factors in males

\begin{tabular}{|c|c|c|c|}
\hline Parameter & $\begin{array}{l}\text { Normal occlusion } \\
\quad(n=628)\end{array}$ & $\begin{array}{l}\text { Crowding } \\
(n=191)\end{array}$ & $P$ value $^{\mathrm{a}}$ \\
\hline $\mathrm{BMI}\left(\mathrm{kg} / \mathrm{m}^{2}\right)$ & $21.3 \pm 3.1$ & $20.7 \pm 3.1$ & 0.016 \\
\hline BMI category & & & 0.001 \\
\hline Underweight $\left(\mathrm{BMI}<18.5 \mathrm{~kg} / \mathrm{m}^{2}\right.$ ) & $92(14.6)$ & $51(26.7)$ & \\
\hline Normal weight (BMI 18.5-24.9kg/m²) & $473(75.3)$ & $121(63.4)$ & \\
\hline Overweight (BMI 25-29.9kg/m²) & $55(8.8)$ & $18(9.4)$ & \\
\hline Obesity $\left(\mathrm{BMI} \geq 30 \mathrm{~kg} / \mathrm{m}^{2}\right.$ ) & $8(1.3)$ & $1(0.5)$ & \\
\hline Number of teeth present & $28.7 \pm 1.3$ & $28.6 \pm 1.2$ & 0.317 \\
\hline Maximum occlusal tooth wear index score & & & 0.129 \\
\hline 0 & $150(23.9)$ & $45(23.6)$ & \\
\hline 1 & $358(57.0)$ & $100(52.4)$ & \\
\hline 2 & $112(17.8)$ & $39(20.4)$ & \\
\hline 3 & $8(1.3)$ & $7(3.7)$ & \\
\hline 4 & $0(0.0)$ & $0(0.0)$ & \\
\hline Buccal mucosa ridging & & & $<0.001$ \\
\hline No & $295(47.0)$ & $58(30.4)$ & \\
\hline Partial & $249(39.6)$ & $94(49.2)$ & \\
\hline Widespread & $84(13.4)$ & $39(20.4)$ & \\
\hline Dental impression on the tongue & $157(25.0)$ & $67(35.1)$ & 0.006 \\
\hline Palatal torus & $4(0.6)$ & $2(1.0)$ & 0.561 \\
\hline Mandibular torus & $80(12.7)$ & $49(25.7)$ & $<0.001$ \\
\hline \multicolumn{4}{|l|}{ Awareness of bruxism } \\
\hline Jaw fatigue on waking in the morning & $15(2.4)$ & $8(4.2)$ & 0.187 \\
\hline Sore teeth or gums on waking in the morning & $12(1.9)$ & $7(3.7)$ & 0.158 \\
\hline Headache on waking in the morning & $46(7.3)$ & $9(4.7)$ & 0.206 \\
\hline Grinding during the day & $10(1.6)$ & $3(1.6)$ & 0.983 \\
\hline Clenching during the day & $26(4.1)$ & $19(9.9)$ & 0.002 \\
\hline Sleep bruxism & $34(5.4)$ & $12(6.3)$ & 0.648 \\
\hline \multicolumn{4}{|l|}{ Oral habits } \\
\hline Biting fingernail/pens/pencils & $67(10.7)$ & $16(8.4)$ & 0.358 \\
\hline Biting mucosa of cheeks/lips & $106(16.9)$ & $44(23.0)$ & 0.054 \\
\hline Gum chewing & $96(15.3)$ & $29(15.2)$ & 0.972 \\
\hline
\end{tabular}

BMI, body mass index.

All values are reported as number (\%) except for BMI, which is reported as mean \pm standard deviation.

at test or $\chi^{2}$ test.

that there is no evidence whatsoever for a causal relationship between bruxism and occlusion. ${ }^{2}$ Therefore, we hypothesized an inverse pathway, in which bruxism may be a risk factor of malocclusion. Our results support this hypothesis. However, the present study was a cross-sectional study; a prospective cohort or intervention study may provide information beyond what we present here.

In the present study, underweight (BMI $<18.5 \mathrm{~kg} / \mathrm{m}^{2}$ ) was also significantly related to malocclusion in males. There is a positive correlation between BMI and cervical vertebral maturation. ${ }^{43}$ Skeletal maturation is also related to malocclusion. ${ }^{44}$ Thus, skeletal prematurity with underweight may be related to malocclusion in males. However, the opposite pathway might be involved in the relationship. Malocclusion negatively affects subjects' ability to process and break down foods, which then attenuates masticatory performance. ${ }^{45}$ In elderly people, masticatory performance is positively related to $\mathrm{BMI} .{ }^{46}$ Malocclusion might induce underweight in young adults via reduction of masticatory performance. Further longitudinal studies are required to investigate the relationship between malocclusion and BMI.

In females, there was no significant association between malocclusion and awareness of bruxism. Although the reason for this lack of observed association is unclear, a possible mechanism may be as follows: in young adults, bite force in males is greater than that in females. ${ }^{4-49}$ Bruxism in females
Table 6. Association between crowding and related factors in females

\begin{tabular}{|c|c|c|c|}
\hline Parameter & $\begin{array}{l}\text { Normal occlusion } \\
\quad(n=394)\end{array}$ & $\begin{array}{l}\text { Crowding } \\
(n=142)\end{array}$ & $P$ value ${ }^{a}$ \\
\hline BMI $\left(\mathrm{kg} / \mathrm{m}^{2}\right)$ & $20.5 \pm 2.4$ & $20.5 \pm 2.5$ & 0.844 \\
\hline BMI category & & & 0.578 \\
\hline Underweight $\left(\mathrm{BMI}<18.5 \mathrm{~kg} / \mathrm{m}^{2}\right)$ & $81(20.6)$ & $29(20.4)$ & \\
\hline Normal weight (BMI 18.5-24.9 kg/m²) & $295(74.9)$ & $109(76.8)$ & \\
\hline Overweight (BMI 25-29.9kg/m²) & $17(4.3)$ & $3(2.1)$ & \\
\hline Obesity $\left(\mathrm{BMI} \geq 30 \mathrm{~kg} / \mathrm{m}^{2}\right)$ & $1(0.3)$ & $1(0.7)$ & \\
\hline Number of teeth present & $28.4 \pm 1.1$ & $28.7 \pm 1.2$ & 0.017 \\
\hline Maximum occlusal tooth wear index score & & & 0.008 \\
\hline 0 & $114(28.9)$ & $44(31.0)$ & \\
\hline 1 & $208(52.8)$ & $60(42.3)$ & \\
\hline 2 & $66(16.8)$ & $29(20.4)$ & \\
\hline 3 & $6(1.5)$ & $9(6.3)$ & \\
\hline 4 & $0(0.0)$ & $0(0.0)$ & \\
\hline Buccal mucosa ridging & & & 0.006 \\
\hline No & $134(34.0)$ & $31(21.8)$ & \\
\hline Partial & $190(48.2)$ & $83(58.5)$ & \\
\hline Widespread & $70(17.8)$ & $28(19.7)$ & \\
\hline Dental impression on the tongue & $118(29.9)$ & $65(45.8)$ & 0.001 \\
\hline Palatal torus & $4(1.0)$ & $8(5.6)$ & 0.001 \\
\hline Mandibular torus & $38(9.6)$ & $40(28.2)$ & $<0.001$ \\
\hline \multicolumn{4}{|l|}{ Awareness of bruxism } \\
\hline Jaw fatigue on waking in the morning & $13(3.3)$ & $7(4.9)$ & 0.380 \\
\hline Sore teeth or gums on waking in the morning & $10(2.5)$ & $4(2.8)$ & 0.858 \\
\hline Headache on waking in the morning & $33(8.4)$ & $16(11.3)$ & 0.305 \\
\hline Grinding during the day & $11(2.8)$ & $6(4.2)$ & 0.403 \\
\hline Clenching during the day & $50(12.7)$ & $17(13.0)$ & 0.824 \\
\hline Sleep bruxism & $37(9.4)$ & $11(7.7)$ & 0.556 \\
\hline \multicolumn{4}{|l|}{ Oral habits } \\
\hline Biting fingernail/pens/pencils & $25(6.3)$ & $8(5.6)$ & 0.762 \\
\hline Biting mucosa of cheeks/lips & $82(20.8)$ & $19(13.4)$ & 0.052 \\
\hline Gum chewing & $28(7.1)$ & $11(7.7)$ & 0.801 \\
\hline
\end{tabular}

BMI, body mass index.

All values are reported as number (\%) except for BMI, which is reported as mean \pm standard deviation.

${ }^{\text {a }} t$ test or $\chi^{2}$ test.

Table 7. Adjusted odds ratios and $95 \%$ confidence intervals for crowding

\begin{tabular}{|c|c|c|c|c|}
\hline Parameter & & Odds ratio & $95 \% \mathrm{Cl}^{\mathrm{a}}$ & $P$ value \\
\hline \multicolumn{5}{|l|}{ Males } \\
\hline \multirow[t]{3}{*}{ BMI category } & $\begin{array}{l}\text { Normal weight } \\
\left(\text { BMI } 18.5-24.9 \mathrm{~kg} / \mathrm{m}^{2} \text { ) }\right.\end{array}$ & 1 & & \\
\hline & $\begin{array}{l}\text { Underweight } \\
\left(\mathrm{BMI}<18.5 \mathrm{~kg} / \mathrm{m}^{2}\right)\end{array}$ & 2.21 & $1.48-3.30$ & $<0.001$ \\
\hline & $\begin{array}{l}\text { Overweight/obesity } \\
\left(\mathrm{BMI} \geq 25 \mathrm{~kg} / \mathrm{m}^{2}\right)\end{array}$ & 1.25 & $0.72-2.18$ & 0.431 \\
\hline $\begin{array}{l}\text { Number of teeth } \\
\text { present }\end{array}$ & & 0.916 & $0.80-1.05$ & 0.194 \\
\hline Clenching & - & 1 & & \\
\hline during the day & + & 2.71 & $1.45-5.07$ & 0.002 \\
\hline \multicolumn{5}{|l|}{ Females } \\
\hline \multirow[t]{3}{*}{ BMI category } & $\begin{array}{l}\text { Normal weight } \\
\left(\text { BMI } 18.5-24.9 \mathrm{~kg} / \mathrm{m}^{2} \text { ) }\right.\end{array}$ & 1 & & \\
\hline & $\begin{array}{l}\text { Underweight } \\
\left(\mathrm{BMI}<18.5 \mathrm{~kg} / \mathrm{m}^{2}\right)\end{array}$ & 1.04 & $0.64-1.69$ & 0.879 \\
\hline & $\begin{array}{l}\text { Overweight/obesity } \\
\left(\mathrm{BMI} \geq 25 \mathrm{~kg} / \mathrm{m}^{2}\right)\end{array}$ & 0.53 & $0.17-1.62$ & 0.266 \\
\hline $\begin{array}{l}\text { Number of teeth } \\
\text { present }\end{array}$ & & 1.24 & $1.06-1.46$ & 0.009 \\
\hline Clenching & - & 1 & & \\
\hline during the day & + & 0.96 & $0.53-1.73$ & 0.886 \\
\hline
\end{tabular}

$\mathrm{BMI}$, body mass index; $\mathrm{Cl}$, confidence interval.

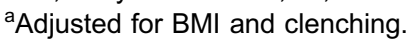

may therefore have smaller effects on tooth movement than that in males because of gender differences in bite force. Thus, in females, the association between bruxism and malocclusion 
Table 8. Association between overjet and related factors in males

\begin{tabular}{|c|c|c|c|}
\hline Parameter & $\begin{array}{l}\text { Normal occlusion } \\
\quad(n=628)\end{array}$ & $\begin{array}{l}\text { Overjet } \\
(n=67)\end{array}$ & $P$ value $^{\mathrm{a}}$ \\
\hline $\mathrm{BMI}\left(\mathrm{kg} / \mathrm{m}^{2}\right)$ & $21.3 \pm 3.1$ & $20.9 \pm 3.0$ & 0.340 \\
\hline BMI category & & & 0.235 \\
\hline Underweight $\left(\mathrm{BMI}<18.5 \mathrm{~kg} / \mathrm{m}^{2}\right)$ & $92(14.6)$ & $16(23.9)$ & \\
\hline Normal weight (BMI $18.5-24.9 \mathrm{~kg} / \mathrm{m}^{2}$ ) & $473(75.3)$ & $46(68.7)$ & \\
\hline Overweight (BMI $25-29.9 \mathrm{~kg} / \mathrm{m}^{2}$ ) & $55(8.8)$ & $4(6.0)$ & \\
\hline Obesity (BMI $\geq 30 \mathrm{~kg} / \mathrm{m}^{2}$ ) & $8(1.3)$ & $1(1.5)$ & \\
\hline Number of teeth present & $28.7 \pm 1.3$ & $28.6 \pm 1.5$ & 0.641 \\
\hline Maximum occlusal tooth wear index score & & & 0.541 \\
\hline 0 & $150(23.9)$ & $12(17.9)$ & \\
\hline 1 & $358(57.0)$ & $38(56.7)$ & \\
\hline 2 & $112(17.8)$ & $16(23.9)$ & \\
\hline 3 & $8(1.3)$ & $1(1.5)$ & \\
\hline 4 & $0(0.0)$ & $0(0.0)$ & \\
\hline Buccal mucosa ridging & & & 0.283 \\
\hline No & $295(47.0)$ & $25(37.3)$ & \\
\hline Partial & $249(39.6)$ & $30(44.8)$ & \\
\hline Widespread & $84(13.4)$ & $12(17.9)$ & \\
\hline Dental impression on the tongue & $157(25.0)$ & $17(25.4)$ & 0.947 \\
\hline Palatal torus & $4(0.6)$ & $0(0.0)$ & 0.512 \\
\hline Mandibular torus & $80(12.7)$ & $12(17.9)$ & 0.235 \\
\hline \multicolumn{4}{|l|}{ Awareness of bruxism } \\
\hline Jaw fatigue on waking in the morning & $15(2.4)$ & $1(1.5)$ & 0.642 \\
\hline Sore teeth or gums on waking in the morning & $12(1.9)$ & $1(1.5)$ & 0.810 \\
\hline Headache on waking in the morning & $46(7.3)$ & $3(4.5)$ & 0.387 \\
\hline Grinding during the day & $10(1.6)$ & $0(0.0)$ & 0.298 \\
\hline Clenching during the day & $26(4.1)$ & $5(7.5)$ & 0.210 \\
\hline Sleep bruxism & $34(5.4)$ & $4(6.0)$ & 0.849 \\
\hline \multicolumn{4}{|l|}{ Oral habits } \\
\hline Biting fingernail/pens/pencils & $67(10.7)$ & $4(6.0)$ & 0.227 \\
\hline Biting mucosa of cheeks/lips & $106(16.9)$ & $16(23.9)$ & 0.152 \\
\hline Gum chewing & $96(15.3)$ & $9(13.4)$ & 0.687 \\
\hline
\end{tabular}

BMI, body mass index.

All values are reported as number (\%) except for BMI, which is reported as mean \pm standard deviation.

at test or $\chi^{2}$ test.

may not have been obvious in this study. However, this possibility cannot explain the fact that malocclusion was more prevalent in females than males, which suggests that there are other risk factors of malocclusion in females, such as genetic factors. Further studies are required to investigate the gender difference.

Malocclusion can induce psychological stress and is associated with self-rated poor oral health in university students. $^{23,37}$ Therefore, evaluation of bruxism might be required in university students for the prevention of malocclusion so that, for people with awareness of clenching, attempts can be made to avoid their clenching habit. In Japanese schools/universities, health examinations are performed on a regular basis, according to school health law. Since early control of the risk factors of malocclusion is essential for prevention in younger populations, monitoring of awareness of bruxism during regular health examinations might be useful.

We also investigated the association between awareness of bruxism and its related clinical factors, including maximum occlusal tooth wear index score, buccal mucosa ridging, tongue indentation, palatal torus, and mandibular torus. Females who had sore teeth and gums or jaw fatigue on waking in the morning had a higher prevalence of palatal torus than those who did not $(12.5 \%$ vs $2.0 \%[P<0.01]$ and $9.5 \%$ vs $2.0 \%[P<0.01]$, respectively). Our results were supported
Table 9. Association between overjet and related factors in females

\begin{tabular}{|c|c|c|c|}
\hline Parameter & $\begin{array}{l}\text { Normal occlusion } \\
\quad(n=394)\end{array}$ & $\begin{array}{l}\text { Overjet } \\
(n=56)\end{array}$ & $P$ value $^{a}$ \\
\hline BMI $\left(\mathrm{kg} / \mathrm{m}^{2}\right)$ & $20.5 \pm 2.4$ & $19.9 \pm 2.0$ & 0.065 \\
\hline BMl category & & & 0.118 \\
\hline Underweight (BMI <18.5 kg/m²) & $81(20.6)$ & $18(32.1)$ & \\
\hline Normal weight (BMI $18.5-24.9 \mathrm{~kg} / \mathrm{m}^{2}$ ) & $295(74.9)$ & $38(67.9)$ & \\
\hline Overweight (BMI 25-29.9 kg/m²) & $17(4.3)$ & $0(0.0)$ & \\
\hline Obesity (BMI $\geq 30 \mathrm{~kg} / \mathrm{m}^{2}$ ) & $1(0.3)$ & $0(0.0)$ & \\
\hline Number of teeth present & $28.4 \pm 1.1$ & $28.2 \pm 1.2$ & 0.297 \\
\hline Maximum occlusal tooth wear index score & & & 0.618 \\
\hline 0 & $114(28.9)$ & $21(37.5)$ & \\
\hline 1 & $208(52.8)$ & $26(46.4)$ & \\
\hline 2 & $66(16.8)$ & $8(14.3)$ & \\
\hline 3 & $6(1.5)$ & $1(1.8)$ & \\
\hline 4 & $0(0.0)$ & $0(0.0)$ & \\
\hline Buccal mucosa ridging & & & 0.298 \\
\hline No & $134(34.0)$ & $14(25.0)$ & \\
\hline Partial & $190(48.2)$ & $33(58.9)$ & \\
\hline Widespread & $70(17.8)$ & $9(16.1)$ & \\
\hline Dental impression on the tongue & $118(29.9)$ & $16(28.6)$ & 0.833 \\
\hline Palatal torus & $4(1.0)$ & $2(3.6)$ & 0.119 \\
\hline Mandibular torus & $38(9.6)$ & $9(16.1)$ & 0.141 \\
\hline \multicolumn{4}{|l|}{ Awareness of bruxism } \\
\hline Jaw fatigue on waking in the morning & $13(3.3)$ & $1(1.8)$ & 0.542 \\
\hline Sore teeth or gums on waking in the morning & $10(2.5)$ & $3(5.4)$ & 0.239 \\
\hline Headache on waking in the morning & $33(8.4)$ & $4(7.1)$ & 0.753 \\
\hline Grinding during the day & $11(2.8)$ & $3(5.4)$ & 0.301 \\
\hline Clenching during the day & $50(12.7)$ & $6(10.7)$ & 0.675 \\
\hline Sleep bruxism & $37(9.4)$ & $4(7.1)$ & 0.584 \\
\hline \multicolumn{4}{|l|}{ Oral habits } \\
\hline Biting fingernail/pens/pencils & $25(6.3)$ & $3(5.4)$ & 0.775 \\
\hline Biting mucosa of cheeks/lips & $82(20.8)$ & $10(17.9)$ & 0.608 \\
\hline Gum chewing & $28(7.1)$ & $5(8.9)$ & 0.625 \\
\hline
\end{tabular}

BMI, body mass index

All values are reported as number (\%) except for BMI, which is reported as mean \pm standard deviation.

${ }^{a} t$ test or $\chi^{2}$ test.

by previous studies. ${ }^{50-52}$ On the other hand, other clinical factors, such as tooth wear, buccal mucosa ridging, tongue indentation, and mandibular torus, were not significantly related to bruxism. Bruxism seems to be one of the contributory factors for the development of tooth wear, oral tori, buccal mucosa ridging, and tongue indentation, and these items are sometimes used for clinical diagnosis of bruxism. ${ }^{41}$ However, most of these items still contain vague factors and their validity for diagnosing bruxism has not been confirmed. ${ }^{41}$

We used a self-administered questionnaire of bruxism because it can be applied to a large population and is convenient for clinicians and researchers to assess the presence or absence of bruxism, especially in epidemiological studies. ${ }^{5,41,53}$ On the other hand, a review suggests that the use of self-reports alone to assess the presence or absence of bruxism is scientifically unreliable. ${ }^{41}$ Actually, the prevalence of bruxism by self-reported questionnaires varies substantially $(4 \%-40 \%){ }^{10}$ which is thought to be due to the limitation of self-reports. ${ }^{41}$ This can be considered a limitation in our study, since we did not measure bruxism activity directly using intra-oral appliances.

Our study has other limitations. First, all participants were recruited from among students at Okayama University, which may limit the ability to extrapolate these findings to the general population of young adults. However, based on 
Ministry of Education, Culture, Sports, Science and Technology in Japan, the proportion of students who went on to universities is about $50 \%$. Further, the prevalence of malocclusion (32.1\%) and awareness of clenching during the day $(8.3 \%)$ were within the ranges reported in previous studies. ${ }^{10,23}$ Thus, there may be potential for generalization. Second, we did not examine other possible confounders, such as non-nutritive sucking, ${ }^{54}$ habitual mouth breathing, ${ }^{55}$ early loss of primary teeth, ${ }^{56}$ genetic factors, and occlusal force. ${ }^{57}$

In conclusion, this study revealed that awareness of clenching and underweight were related to malocclusion (crowding) in university male students. These findings suggest that clenching and BMI may be considered in screening for malocclusion risk in young male adults.

\section{ONLINE ONLY MATERIAL}

Abstract in Japanese.

\section{ACKNOWLEDGMENTS}

Conflicts of interest: None declared.

\section{REFERENCES}

1. The glossary of prosthodontic terms. J Prosthet Dent. 2005;94:10-92.

2. Lobbezoo F, Ahlberg J, Manfredini D, Winocur E. Are bruxism and the bite causally related? J Oral Rehabil. 2012;39:489-501.

3. American Academy of Sleep Medicine. International classification of sleep disorders, 2nd ed. Westchester: American Academy of Sleep Medicine; 2005.

4. Lobbezoo F, Aarab G, van der Zaag J. Definitions, epidemiology, and etiology of sleep bruxism. In: Lavigne GJ, Cistulli P, Smith M, editors. Sleep medicine for dentists: a practical overview. Chicago: Quintessence Publishing Co, Inc; 2009. p. 95-100.

5. Paesani DA. Bruxism theory and practice. Chicago: Quintessence Publishing Co, Inc; 2010.

6. Lavigne GJ, Montplaisir JY. Restless legs syndrome and sleep bruxism: prevalence and associations among Canadians. Sleep. 1994;17:739-43.

7. Ohayon MM, Li KK, Guilleminault C. Risk factors for sleep bruxism in the general population. Chest. 2001;119:53-61.

8. Attanasio R. Intraoral orthotic therapy. Dent Clin North Am. 1997;41:309-24.

9. Attanasio R. Nocturnal bruxism and its clinical management. Dent Clin North Am. 1991;35:245-52.

10. Seligman DA, Pullinger AG, Solberg WK. The prevalence of dental attrition and its association with factors of age, gender, occlusion, and TMJ symptomatology. J Dent Res. 1988;67: 1323-33.

11. van Selms MK, Visscher CM, Naeije M, Lobbezoo F. Bruxism and associated factors among Dutch adolescents. Community Dent Oral Epidemiol. 2013;41:353-63.

12. Strausz T, Ahlberg J, Lobbezoo F, Restrepo CC, Hublin C,
Ahlberg K, et al. Self-reported bruxism from adolescence to young adulthood: a nine-year follow-up. J Oral Rehabil. 2010;37:497-500.

13. Lobbezoo F, Naeije M. Bruxism is mainly regulated centrally, not peripherally. J Oral Rehabil. 2001;28:1085-91.

14. Lobbezoo F, Van Der Zaag J, Naeije M. Bruxism: its multiple causes and its effects on dental implants. An updated review. J Oral Rehabil. 2006;33:293-300.

15. Pierce CJ, Chrisman K, Bennett ME, Close JM. Stress, anticipatory stress, and psychologic measures related to sleep bruxism. J Orofac Pain. 1995;9:51-6.

16. Manfredini D, Lobbezoo F. Role of psychosocial factors in the etiology of bruxism. J Orofac Pain. 2009;23:153-66.

17. Lobbezoo F, Soucy JP, Montplaisir JY, Lavigne GJ. Striatal D2 receptor binding in sleep bruxism: a controlled study with iodine-123-iodobenzamide and single photon emission computed tomography. J Dent Res. 1996;75:1804-10.

18. Lobbezoo F, Lavigne GJ, Tanguay R, Montplaisir JY. The effect of the catecholamine precursor L-dopa on sleep bruxism: a controlled clinical trial. Mov Disord. 1997;12:73-8.

19. Lobbezoo F, Soucy JP, Hartman NG, Montplaisir JY, Lavigne GJ. Effects of the dopamine D2 receptor agonist bromocriptine on sleep bruxism: report of two single-patient clinical trials. J Dent Res. 1997;76:1610-4.

20. Hublin C, Kaprio J, Partinen M, Koskenvuo M. Sleep bruxism on self-report in a nationwide twin cohort. J Sleep Res. 1998;7(1):61-7.

21. Lavigne GL, Lobbezoo F, Rompré PH, Nielsen TA, Montplaisir J. Cigarette smoking as a risk or exacerbating factor for restless legs syndrome and sleep bruxism. Sleep. 1997;20:290-3.

22. Johansson A, Omar R, Carlsson GE. Bruxism and prosthetic treatment: a critical review. J Prosthodont Res. 2011;55:127-36.

23. Ekuni D, Furuta M, Irie K, Azuma T, Tomofuji T, Murakami T, et al. Relationship between impacts attributed to malocclusion and psychological stress in young Japanese adults. Eur J Orthod. 2011;33:558-63.

24. Vig KW, Fields HW. Facial growth and management of orthodontic problems. Pediatr Clin North Am. 2000;47: 1085-123.

25. Macena MC, Katz CR, Rosenblatt A. Prevalence of a posterior crossbite and sucking habits in Brazilian children aged 18-59 months. Eur J Orthod. 2009;31:357-61.

26. Moimaz SA, Garbin AJ, Lima AM, Lolli LF, Saliba O, Garbin CA. Longitudinal study of habits leading to malocclusion development in childhood. BMC Oral Health. 2014;14:96.

27. Abboud M, Grüner M, Koeck B. Anterior crowding-just an esthetic problem? J Orofac Orthop. 2002;63:264-73.

28. Shetty S, Pitti V, Satish Babu CL, Surendra Kumar GP, Deepthi BC. Bruxism: a literature review. J Indian Prosthodont Soc. 2010;10:141-8.

29. Pintado MR, Anderson GC, DeLong R, Douglas WH. Variation in tooth wear in young adults over a two-year period. J Prosthet Dent. 1997;77:313-20.

30. Mizutani S, Ekuni D, Tomofuji T, Azuma T, Irie K, Machida T, et al. Factors related to the formation of buccal mucosa ridging in university students. Acta Odontol Scand. 2014;72:58-63.

31. Emodi-Perlman A, Eli I, Friedman-Rubin P, Goldsmith C, Reiter $\mathrm{S}$, Winocur E. Bruxism, oral parafunctions, anamnestic and 
clinical findings of temporomandibular disorders in children. J Oral Rehabil. 2012;39:126-35.

32. Panek H, Nawrot P, Mazan M, Bielicka B, Sumisławska M, Pomianowski R. Coincidence and awareness of oral parafunctions in college students. Community Dent Health. 2012;29:74-7.

33. Gavish A, Halachmi M, Winocur E, Gazit E. Oral habits and their association with signs and symptoms of temporomandibular disorders in adolescent girls. J Oral Rehabil. 2000;27:22-32.

34. Yamane M, Ekuni D, Mizutani S, Kataoka K, SakumotoKataoka M, Kawabata Y, et al. Relationships between eating quickly and weight gain in Japanese university students: A longitudinal study. Obesity (Silver Spring). 2014;22:2262-6.

35. World Health Organization. Obesity: Preventing and managing the global epidemic. Report of a WHO Consultation on Obesity; Geneva, p. 3-5; 1997. Geneva: WHO.

36. Smith BG, Knight JK. An index for measuring the wear of teeth. Br Dent J. 1984;156:435-8.

37. Kojima A, Ekuni D, Mizutani S, Furuta M, Irie K, Azuma T, et al. Relationships between self-rated oral health, subjective symptoms, oral health behavior and clinical conditions in Japanese university students: a cross-sectional survey at Okayama University. BMC Oral Health. 2013;13:62.

38. Burden DJ, Pine CM, Burnside G. Modified IOTN: an orthodontic treatment need index for use in oral health surveys. Community Dent Oral Epidemiol. 2001;29:220-5.

39. Yoshinaka M, Ikebe K, Furuya-Yoshinaka M, Hazeyama T, Maeda Y. Prevalence of torus palatinus among a group of Japanese elderly. J Oral Rehabil. 2010;37:848-53.

40. Jainkittivong A, Apinhasmit W, Swasdison S. Prevalence and clinical characteristics of oral tori in 1520 Chulalongkorn University Dental School patients. Surg Radiol Anat. 2007;29: 125-31.

41. Koyano K, Tsukiyama Y, Ichiki R, Kuwata T. Assessment of bruxism in the clinic. J Oral Rehabil. 2008;35:495-508.

42. Oh SH, Nakano M, Bando E, Shigemoto S, Kori M. Evaluation of proximal tooth contact tightness at rest and during clenching. J Oral Rehabil. 2004;31:538-45.

43. Mack KB, Phillips C, Jain N, Koroluk LD. Relationship between body mass index percentile and skeletal maturation and dental development in orthodontic patients. Am J Orthod Dentofacial Orthop. 2013;143:228-34.

44. Armond MC, Generoso R, Falci SG, Ramos-Jorge ML, Marques LS. Skeletal maturation of the cervical vertebrae: association with various types of malocclusion. Braz Oral Res. 2012;26:
145-50.

45. English JD, Buschang PH, Throckmorton GS. Does malocclusion affect masticatory performance? Angle Orthod. 2002;72:21-7.

46. Moriya S, Notani K, Murata A, Inoue N, Miura H. Analysis of moment structures for assessing relationships among perceived chewing ability, dentition status, muscle strength, and balance in community-dwelling older adults. Gerodontology. 2014;31(4): 281-7.

47. Koç D, Doğan A, Bek B. Effect of gender, facial dimensions, body mass index and type of functional occlusion on bite force. J Appl Oral Sci. 2011;19:274-9.

48. Varga S, Spalj S, Lapter Varga M, Anic Milosevic S, Mestrovic S, Slaj M. Maximum voluntary molar bite force in subjects with normal occlusion. Eur J Orthod. 2011;33:427-33.

49. Bonakdarchian M, Askari N, Askari M. Effect of face form on maximal molar bite force with natural dentition. Arch Oral Biol. 2009;54:201-4.

50. Kerdpon D, Sirirungrojying S. A clinical study of oral tori in southern Thailand: prevalence and the relation to parafunctional activity. Eur J Oral Sci. 1999;107:9-13.

51. Restrepo CC, Sforza C, Colombo A, Peláez-Vargas A, Ferrario VF. Palate morphology of bruxist children with mixed dentition. A pilot study. J Oral Rehabil. 2008;35:353-60.

52. Morrison MD, Tamimi F. Oral tori are associated with local mechanical and systemic factors: a case-control study. J Oral Maxillofac Surg. 2013;71:14-22.

53. Okeson JP, Phillips BA, Berry DT, Cook Y, Paesani D, Galante J. Nocturnal bruxing events in healthy geriatric subjects. J Oral Rehabil. 1990;17:411-8.

54. Kasparaviciene K, Sidlauskas A, Zasciurinskiene E, Vasiliauskas A, Juodzbalys G, Sidlauskas M, et al. The prevalence of malocclusion and oral habits among 5-7-year-old children. Med Sci Monit. 2014;20:2036-42.

55. Souki BQ, Pimenta GB, Souki MQ, Franco LP, Becker HM, Pinto JA. Prevalence of malocclusion among mouth breathing children: do expectations meet reality? Int $\mathrm{J}$ Pediatr Otorhinolaryngol. 2009;73:767-73.

56. Grippaudo C, Pantanali F, Paolantonio EG, Grecolini ME, Saulle $\mathrm{R}$, La Torre $\mathrm{G}$, et al. Prevalence of malocclusion in Italian schoolchildren and orthodontic treatment need. Eur J Paediatr Dent. 2013;14:314-8.

57. Ting TY, Wong RW, Rabie AB. Analysis of genetic polymorphisms in skeletal Class I crowding. Am J Orthod Dentofacial Orthop. 2011;140:e9-15. 